\title{
Posterior Neck Triangle Spindle Cell Lipoma
}

\author{
Ali A. Salamat ${ }^{\mathrm{a}, \mathrm{b}, \mathrm{c}}$, Tom Paterson ${ }^{\mathrm{a}}$, Nimesh N. Patel ${ }^{\mathrm{a}}$, \\ Tahwinder Singh ${ }^{\mathrm{a}}$
}

\begin{abstract}
Spindle cell lipomas (SCLs) are a rare variant of lipomas with distinct pathological and immunohistological features. Pathological characteristics of SCLs have been well described and include the following features: a well-circumscribed subcutaneous lesion that contains a variety of mature adipocytes, spindle cells and collagen bundles. Immunohistochemical staining of SCLs is usually positive for CD34. Four cases of posterior neck triangle SCLs have been reported in the literature. We present a further case of SCL presenting in the posterior triangle of the neck. A 51-year-old man with a large right posterior neck triangle mass was referred to our Head \& Neck Clinic. Further questioning revealed that this swelling had been growing over a two and a half year period associated with neck pain, difficulty swallowing and recurrent skin infections at the base of the mass. Due to the rare nature of SCLs, such cases should be discussed in a dedicated multi-disciplinary team meeting. Although metastasis from SCLs is rare, experienced pathologists and radiologists should be consulted early in order to distinguish the type of lesion and the risk of metastasis from more sinister lesions such as liposarcoma. Surgical excision of SCLs is currently accepted as the gold standard and mainstay of management.
\end{abstract}

Keywords: Spindle cell lipoma; Posterior neck triangle; CD34

\section{Introduction}

Spindle cell lipomas (SCLs) are a rare variant of lipomas with distinct pathological and immunohistological features. Enzinger and Harvey first described SCLs as a benign lesion occurring in middle-aged men [1]. A number of anatomical sites affected by this disease have been reported in the literature including the head and neck, oral cavity, orbit, upper back, abdo-

Manuscript accepted for publication March 16, 2016

aDepartment of Otolaryngology/Head \& Neck Surgery, University Hospital Southampton NHS Foundation Trust, Southampton, UK

${ }^{b}$ Academic Unit of Clinical and Experimental Sciences, University of Southampton Faculty of Medicine, Southampton, UK

${ }^{\mathrm{c} C}$ Corresponding Author: Ali A. Salamat, LE57 South Academic Block, University Hospital Southampton NHS Foundation Trust, Tremona Road, Southampton, Hampshire, SO166YD, UK. Email: alisalamat@doctors.org.uk

doi: http://dx.doi.org/10.14740/jmc2462w men, esophagus, breast, bronchus, vulva and extremities.

Pathological characteristics of SCLs have been well described and include the following features: a well-circumscribed subcutaneous lesion that contains a variety of mature adipocytes, spindle cells and collagen bundles [1-3].

Immunohistochemical staining of SCLs is usually positive for CD34. However, this is not pathognomonic and is used as an aid for making a diagnosis [2, 4].

Four cases of posterior neck triangle SCLs have been reported in the literature [5-8]. We present a further case of SCL presenting in the posterior triangle and discuss the current pathological methods used to diagnose SCLs and the surgical approach taken to manage such cases.

\section{Case Report}

A 51-year-old man with a large right posterior neck triangle mass was referred to our Head \& Neck Clinic. Further questioning revealed that this swelling had been growing for over two and a half years. An increased rate of growth and associated symptoms (neck pain, difficulty swallowing and recurrent skin infections at the base of the mass) over the preceding months led the patient to present for medical attention. Functionally, the patient was experiencing great difficulty washing and dressing and therefore was eager to have the lesion removed.

The patient was born prematurely at 34 weeks of age and subsequently developed hydrocephalus requiring a VP shunt system. His other comorbidities included epileptic seizures, chronic calcified subdural hemorrhages, gastroesphageal reflux disease, inflammatory bowel syndrome and osteoarthritis. He was a life long non-smoker on a number of medications to control his seizures and reflux disease.

\section{Radiology}

Ultrasound imaging was undertaken in the first instance. A well-circumscribed mass on the right posterior neck triangle was visualized. No areas of internal vascularity were noted. An ultrasound-guided core biopsy was performed.

Computed tomography (CT) imaging was performed revealing a fat encapsulated mass in the right posterior triangle measuring $106 \times 72 \times 92 \mathrm{~mm}$. The posterior edge of the right sternocleidomastoid muscle was displaced laterally. No internal calcification, cervical lymphadenopathy or metastatic le- 


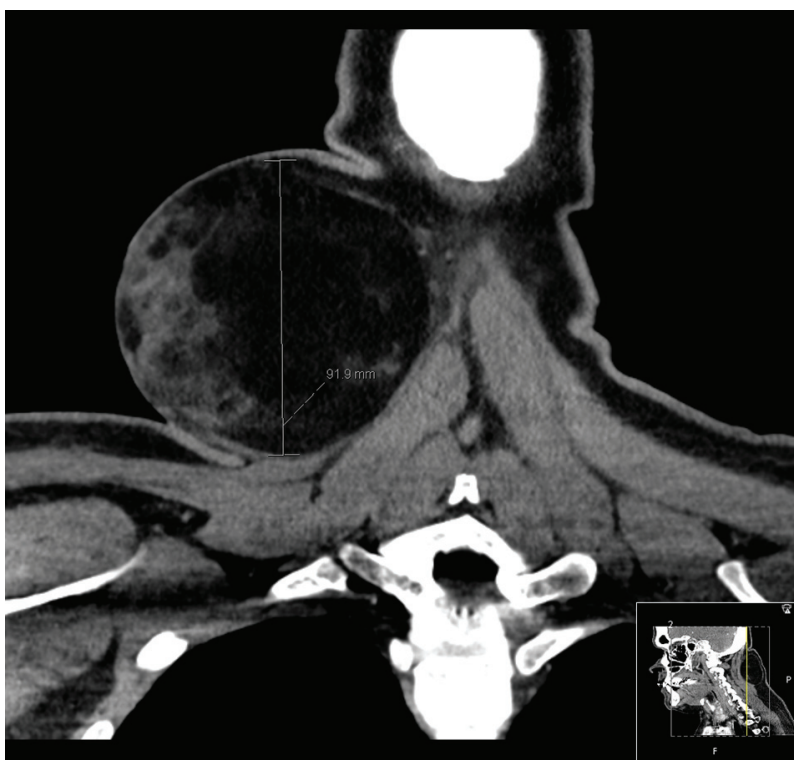

Figure 1. Coronal CT of a large right posterior neck triangle mass abutting the sternocleidomastoid muscle.

sions were identified (Figs. 1-3). Following discussion at the multi-disciplinary team meeting, magnetic resonance imaging (MRI) was recommended. Unfortunately, MRI was not possible as the patient was unable to tolerate lying flat for a period of time needed for the study.

\section{Surgery}

Following general anesthesia and local infiltration with lignocaine and adrenaline, the patient was placed in the left lateral position for best exposure (Figs. 4 and 5). An elliptical cervical incision was made. A posterior neck dissection was

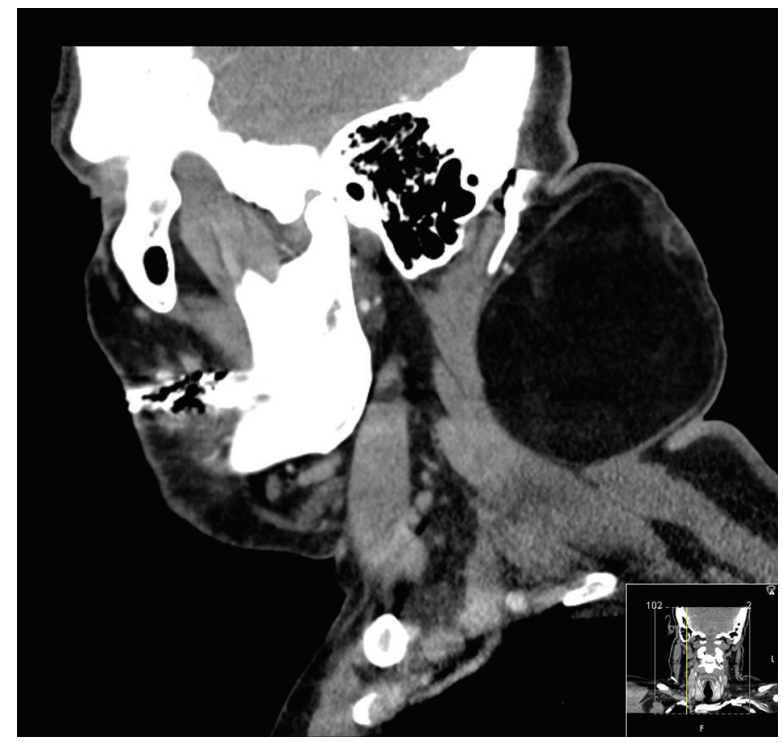

Figure 2. Sagittal CT image delineating the dimensions of the tumor.

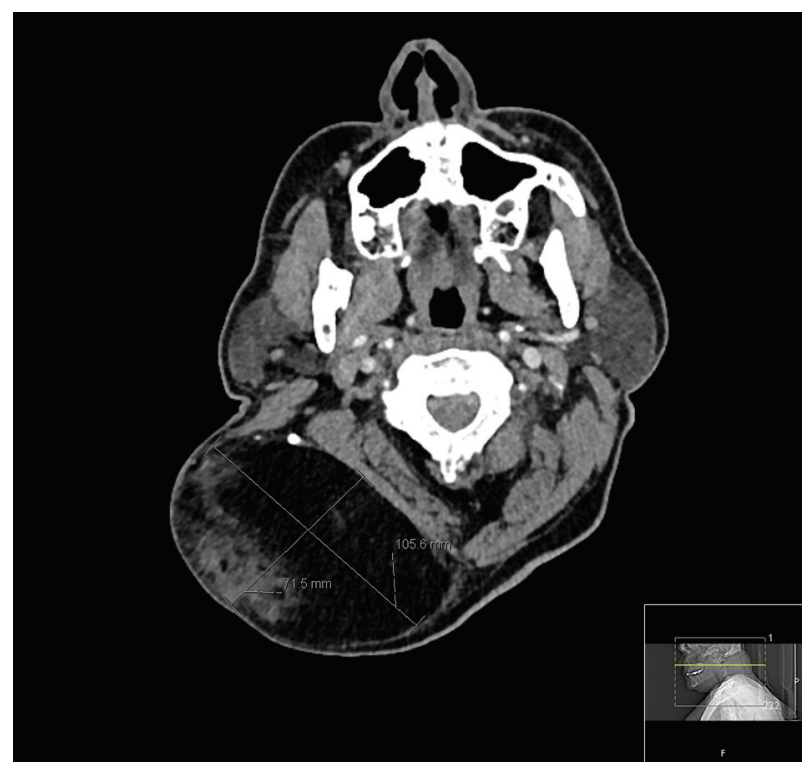

Figure 3. Axial CT image delineating the dimensions of the tumor.

performed using a harmonic scalpel to facilitate a bloodless field, en bloc resection and an adequate margin as described in the literature $[9,10]$. The accessory nerve was subsequently identified and preserved with the aid of a nerve stimulator. Deeper dissection of the lesion revealed an outer appearance of a pseudocapsule (Fig. 6), in addition to its infiltrative nature abutting the posterior edge of the right sternocleidomastoid muscle.

Enlarged branches of the deep cervical, occipital and posterior auricular arteries were encountered and ligated. The lesion was removed with an ellipse of overlying skin to enable closure and satisfactory cosmesis (Fig. 7). A drain was placed overnight, and subsequently removed the following day prior to discharge. At 2-month follow-up, the patient made an excellent recovery, reporting no complications from surgery and expressed his satisfaction with wound healing and cosmesis (Fig. 8).

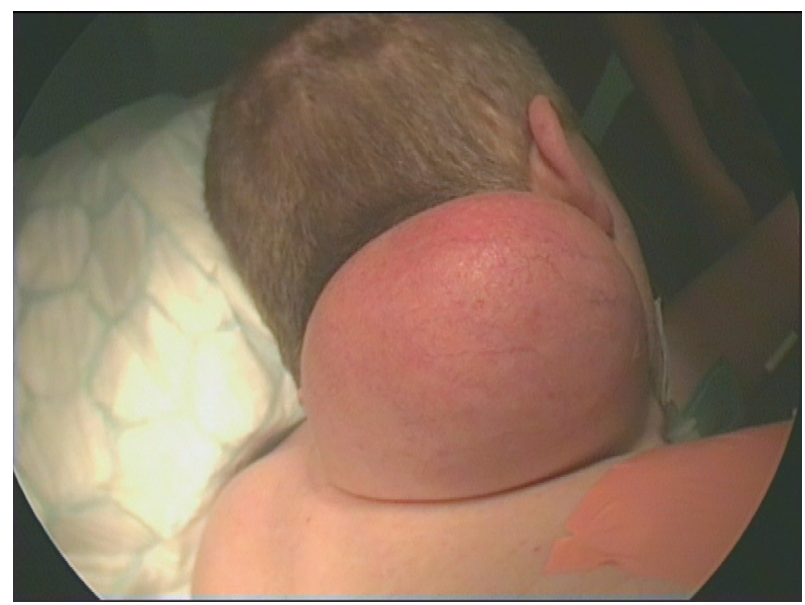

Figure 4. Pre-operative photograph of a large right posterior neck mass. 


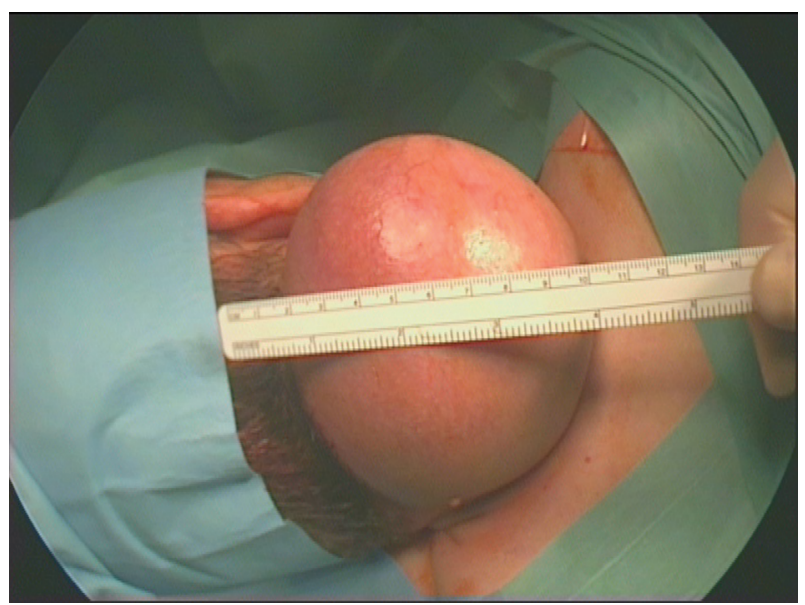

Figure 5. Pre-operative photograph of a large right posterior neck mass (draped).

\section{Pathological analysis}

The $1.2 \mathrm{~kg}$ lesion following microscopic histopathological analysis revealed a completely excised, well-circumscribed and encapsulated tumor composed of mature adipocytes interspersed with fascicles of bland spindle cells (Figs. 9 and 10). Immunochemistry confirmed spindle cells to be positive for CD34 (Fig. 11). Molecular genetic studies in the form of $\mathrm{Mdm} 2$ gene amplification were undertaken but were negative when assessed with fluorescence in situ hybridization imaging. These findings were consistent with a diagnosis of an SCL.

\section{Discussion}

SCLs represent a small and rare subset of lipomas in general. To the best of our knowledge, there have been four other reports documenting posterior neck triangle SCLs. Therefore, this report represents the fifth documented case of a posterior

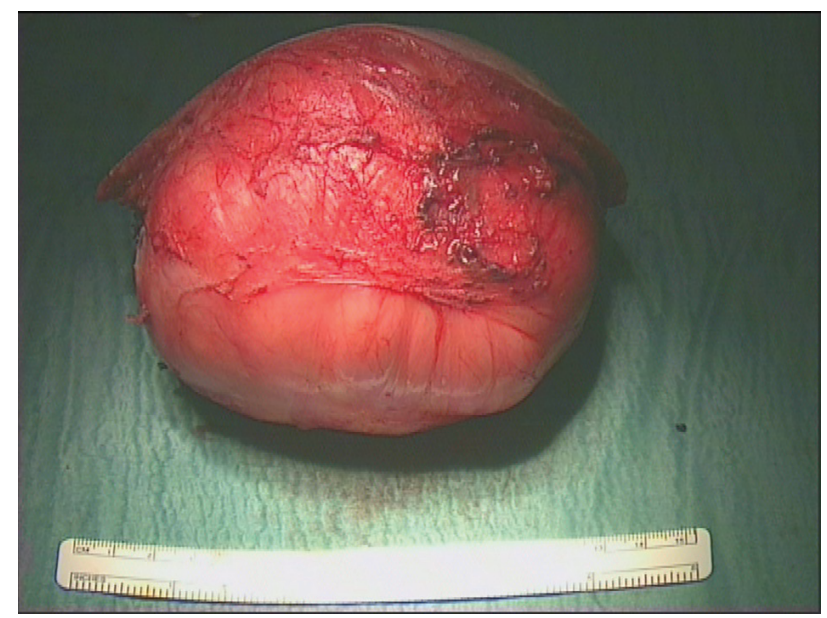

Figure 6. Lesion following complete resection illustrating intact capsule.

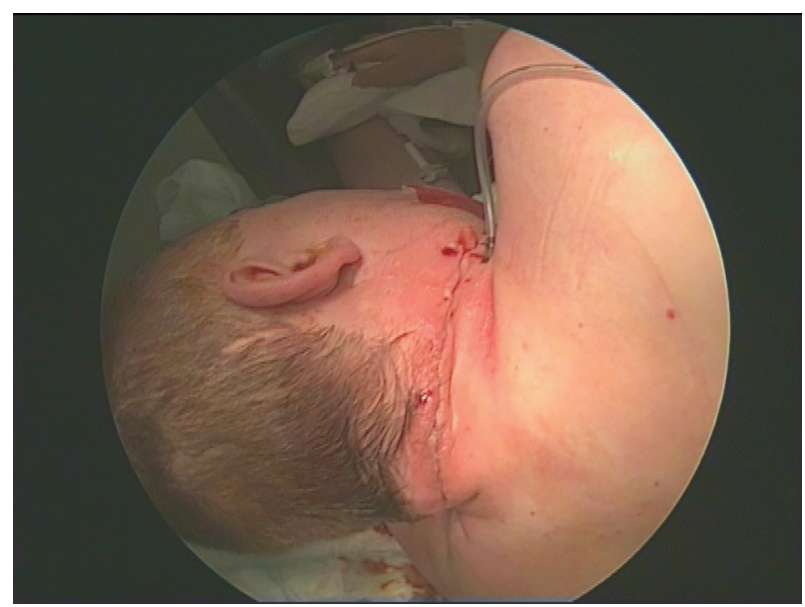

Figure 7. Post-operative appearances following closure.

neck SCL.

SCLs have the tendency of presenting as a solitary, painless, slow growing, benign lesion presenting on the posterior neck or upper back of middle-aged men [3,11]. Metastasis of this lesion is uncommon and has not been reported in the literature.

Following clinical examination, pathological analysis and radiological imaging are necessary to aid diagnosis and formulate a plan for surgical excision. Reoccurrence following surgical excision is thought to be low.

Light microscopic appearances of SCLs consist of mature adipocytes either partially or fully substituted by a homogeneous group of spindle cells [3].

Further definition of an SCL is they are atypical benign lesions, composed of atypical adipocytes with varying shaped and sized hyperchromatic nuclei associated with slightly atypical spindle-shaped cells [4].

The growth of SCLs is somewhat similar to Schwannomas and neurofibromas; however, precise histological analysis would sufficiently differentiate the latter diseases from SCL [3].

CD34 expression is not pathognomonic for a diagnosis of SCL. CD34 immunoreactivity can also be demonstrated in

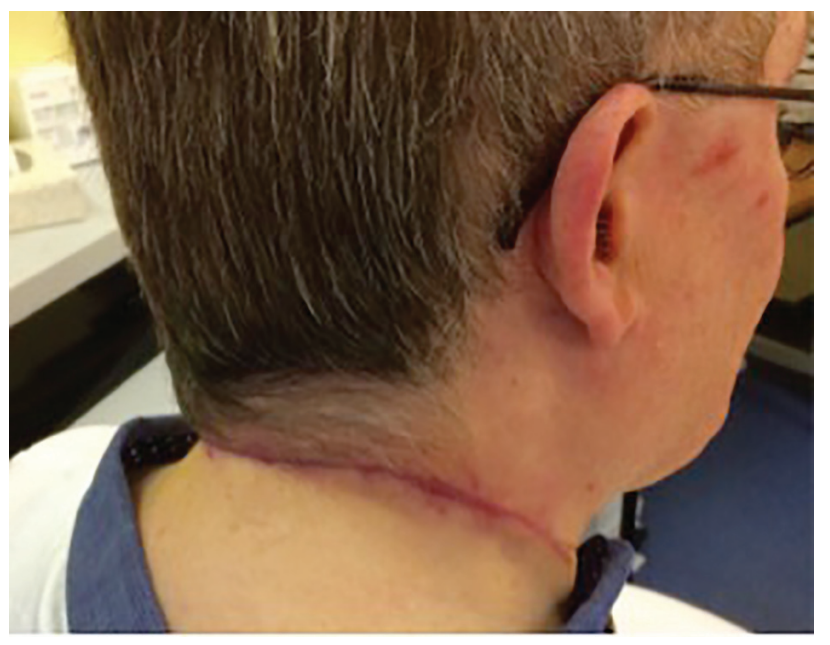

Figure 8. Appearance of the wound 2 months post-operatively. 


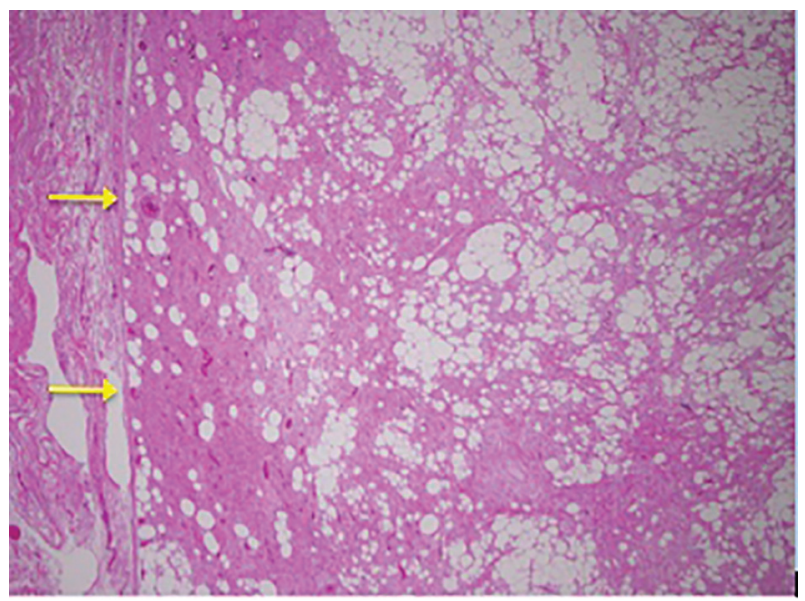

Figure 9. Low power image showing capsule of lesion on left.

atypical lipomatous neoplasms and well-differentiated liposarcomas $[4,12]$. Therefore, CD34 expression alone is not pathologically diagnostic of SCL, but can aid the pathologist in making the diagnosis. This is vital, as low-grade liposarcomas carry a significant risk of metastasis.

SCLs are associated with negative Mdm2 and/or CDK4 gene immunohistochemical expression. It is thought that although some SCLs may express Mdm2 or CDK4 on immunohistochemical staining, invariably, such expression is not detected using fluorescence in situ hybridization (FISH) analysis which is considered to be the gold standard and more sensitive in detecting such gene expression [4].

Radiological appearances supportive of a diagnosis of SCL include a lesion consisting of $25-75 \%$ of fat in the form of adipose globules containing a variable amount of thin and thick septa [11]. Signal intensity of the non-adipose component of SCLs is greater than fat on conventional T2-weighted images, whilst gadolinium-enhancement MRI would show enhancement similar to that of the adjacent vessels [11]. Vascular prominence of the non-adipose tissue contained within SCLs is considered to be significantly more intense than liposarco-

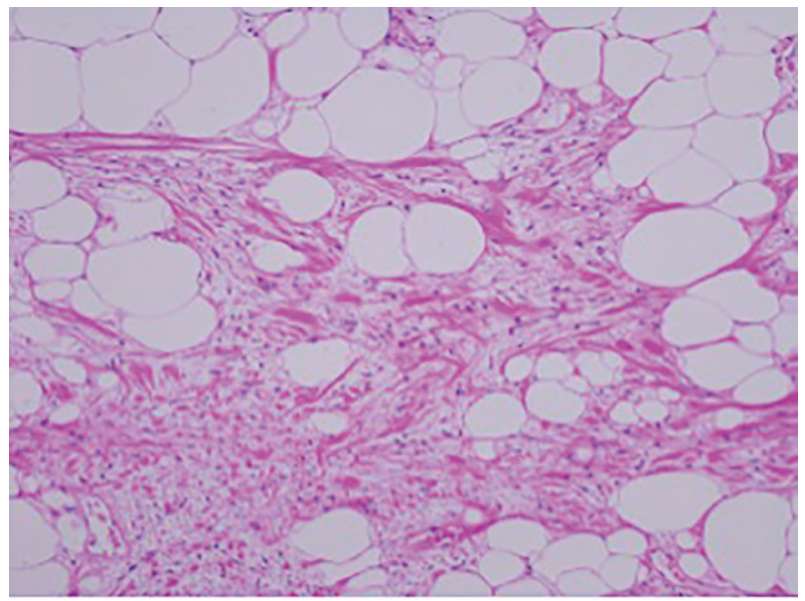

Figure 10. Magnification image $(\times 10)$ showing spindle cells admixed with ropey collagen and mature adipocytes in a myxoid matrix.

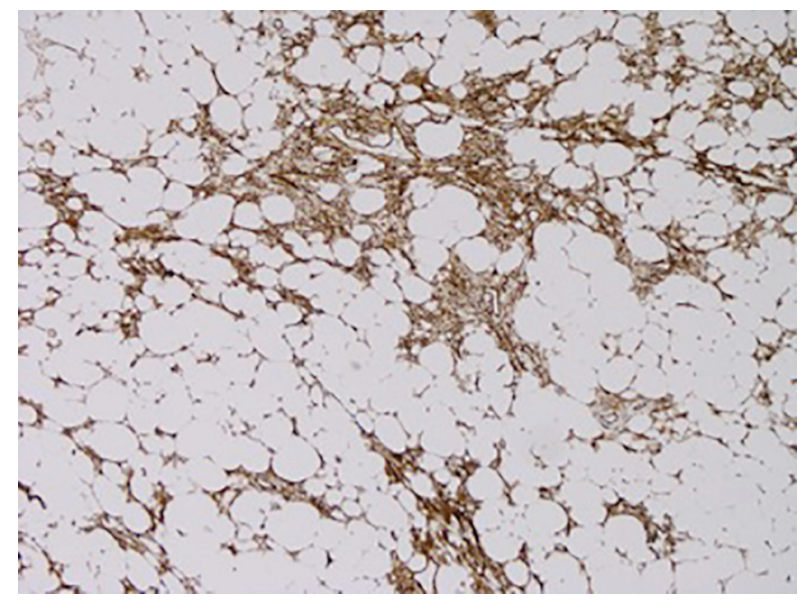

Figure 11. CD34 immunostain showing positivity in the spindle cell areas (brown coloration).

ma. However, calcification and bony erosion are not associated with SCLs on imaging [11].

Due to the rare nature of SCLs, such cases should be discussed in a dedicated multi-disciplinary team meeting. Although metastasis from SCLs is rare, experienced pathologists and radiologists should be consulted early in order to distinguish the type of lesion and the risk of metastasis from more sinister lesions such as liposarcoma. Surgical excision of SCLs is currently accepted as the gold standard and mainstay of management.

\section{Conclusion}

SCLs are a rare, slow growing and benign form of the lipoma family. They tend to present in middle-aged men usually on the upper back or posterior neck. Although the risk of metastasis is low, early and definitive diagnosis is essential in order to rule out lipomas with a higher incidence of metastasis. This is the fifth case to report a posterior neck SCL. Similar to the previous cases, surgical excision was undertaken, which is considered to be the gold standard treatment.

\section{Acknowledgments}

We thank Dr Charles Tilley, Consultant Histopathologist at University Hospital Southampton NHS Foundation Trust for preparing the pathology pictures provided in this report.

\section{Conflicts of Interest}

The authors declare that no competing interests exist.

\section{Grants}

None. 


\section{References}

1. Enzinger FM, Harvey DA. Spindle cell lipoma. Cancer. 1975;36(5):1852-1859.

2. Ide H, Nakagawa T, Kamiyama Y, Muto S, Imamura T, Horie S. Spindle cell lipoma of the spermatic cord. Int J Urol. 2007;14(11):1046-1047.

3. Bolen JW, Thorning D. Spindle-cell lipoma. A clinical, light- and electron-microscopical study. Am J Surg Pathol. 1981;5(5):435-441.

4. Mentzel T, Palmedo G, Kuhnen C. Well-differentiated spindle cell liposarcoma ('atypical spindle cell lipomatous tumor') does not belong to the spectrum of atypical lipomatous tumor but has a close relationship to spindle cell lipoma: clinicopathologic, immunohistochemical, and molecular analysis of six cases. Mod Pathol. 2010;23(5):729-736.

5. Eryilmaz M, Yigit T, Ozturk G, Safali M, Aksu AY. Giant spindle cell lipoma of the posterior neck. Dermatol Surg. 2007;33(10):1258-1261.

6. Senen D, Adanali G, Sevin A, Deren O, Erdogan B. Buffalo hump: spindle cell lipoma in posterior part of the neck. Dermatol Surg. 2007;33(1):73-75.

7. Petit D, Menei P, Fournier HD. An unusual and spectacular case of spindle cell lipoma of the posterior neck invading the spinal cervical canal and posterior cranial fossa. $\mathrm{J}$ Neurosurg Spine. 2011;15(5):502-506.

8. Ghazanfari A, Oppenheimer R. Massive spindle cell lipoma. Otolaryngol Head Neck Surg. 2006;134(1):164-165.

9. Upile T, Jerjes W, Nouraei SA, Singh S, Clarke P, RhysEvans P, Hopper C, et al. How we do it: a method of neck dissection for histopathological analysis. BMC Surg. 2007;7:21.

10. Upile T, Fisher C, Jerjes W, El Maaytah M, Searle A, Archer D, Michaels L, et al. The uncertainty of the surgical margin in the treatment of head and neck cancer. Oral Oncol. 2007;43(4):321-326.

11. Bancroft LW, Kransdorf MJ, Peterson JJ, Sundaram $\mathrm{M}$, Murphey $\mathrm{MD}$, O'Connor MI. Imaging characteristics of spindle cell lipoma. AJR Am J Roentgenol. 2003;181(5):1251-1254.

12. Olaleye O, Fu B, Moorthy R, Lawson C, Black M, Mitchell D. Left supraclavicular spindle cell lipoma. Int J Otolaryngol. 2010;2010:942152. 